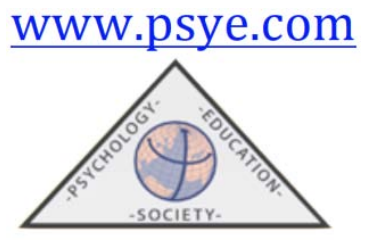

(C) Psychology, Society, \& Education, 2017. Vol. 9(1), pp. 1-10

ISSN 2171-2085 (print) / ISSN 1989-709X (online)

\title{
Enseñanza y Aprendizaje de las Matemáticas: La necesidad de un análisis multidisciplinar
}

\author{
Gamal CERDA*; Carlos PÉREZ*; José A. CASAS ${ }^{\text {1***}}$ y Rosario ORTEGA-RUIZ*** \\ ${ }^{*}$ University of Concepción, Chile ${ }^{* *}$ University of O’Higgins, Chile. ${ }^{* * *}$ University of Córdoba, España
}

(Recibido 27 Mayo, 2016; Aceptado 16 Junio, 2016)

RESUMEN: El logro y aprendizaje exitoso en matemáticas sigue siendo un desafío a nivel mundial, y se realizan grandes esfuerzos para avanzar en propuestas de mejora, generalmente involucran al profesorado, en términos de su formación, perfeccionamiento o práctica de aula. Los esfuerzos también se orientan hacia el examen del rol de diversas variables o factores que pueden predecir o relacionarse con un mejor nivel de aprovechamiento de esta materia escolar. Todo ello revela el carácter complejo y multifactorial de la enseñanza y aprendizaje de las matemáticas, que obliga a los diversos agentes educativos e investigadores a avanzar y profundizar aún más en su estudio. Con la finalidad de contribuir al examen crítico y empírico de algunas variables contextuales o socio cognitivas asociadas al aprendizaje de las matemáticas o la resolución de problemas, como también al examen de algunas propuestas de innovación en el área y la evaluación de su impacto en algunas competencias o habilidades matemáticas, se presenta un conjunto de trabajos derivados de la investigación de grupos de investigación de amplia y destacada trayectoria en el área. El presente número monográfico de la revista Psychology, Society and Education, reúne un total de diez artículos cuyo objetivo principal es contribuir con evidencia y alternativas de análisis a un área del aprendizaje escolar asociada generalmente con altos niveles de fracaso y emociones negativas.

Palabras clave: Matemáticas, aprendizaje, habilidades matemáticas, innovación.

Teaching and Learning of Mathematics: The Need for a Multidisciplinary Analysis

ABSTRACT: Success in learning mathematics remains a global challenge, and great efforts are being made to advance proposals for improvement, generally involving teachers in terms of their training, improvement or classroom practice. Efforts are also oriented toward examining the role of various variables or factors that can predict or relate to a better level of achievement of this school subject. All this reveals the complex and multifactorial nature of the teaching and learning of mathematics, which obliges the various educational agents and researchers to go further and deepen their study. In order to contribute to the critical and empirical examination of some contextual or socio-cognitive variables associated with learning mathematics or problem solving, as well as to the examination of some innovation proposals in the area and the evaluation of their impact on some competences Or mathematical skills, a set of papers derived from the research of research groups of wide and outstanding trajectory in the area is presented. The present issue of the journal Psychology, Society and Education, brings together a total of ten articles whose main objective is to contribute evidence and analysis alternatives to an area of school learning generally associated with high levels of failure and negative emotions. 
Keywords: Mathematics, learning, mathematical skills, innovation.

${ }^{1}$ Correspondencia: Universidad de Córdoba, Facultad Ciencias de la Educación. Avda. San Alberto Magno s/n, 14004, Córdoba. E-mail: jacasas@uco.es.

En todo sistema educativo formal está presente la asignatura de matemáticas en el currículo. Casi no importa el país que se visite, el currículo considera relevante el aprendizaje de esta disciplina. A pesar de esta intención consensuada, tal acuerdo deja de existir al momento de resolver cuál podría ser la mejor manera de poder llevar a cabo el proceso de enseñanza-aprendizaje de esta disciplina en el aula, pues en dicho proceso intervienen múltiples factores, como por ejemplo, la formación que tengan los profesores, su seguridad sobre la disciplina, la didáctica utilizada en el aula, la autonomía de trabajo, el nivel cultural de los padres, el clima escolar y propuesta educativa del establecimiento, por mencionar algunos. Hay abundante literatura que muestra efectos directos, indirectos, individuales y conjuntos de estos y otros factores en el estudio de las matemáticas escolares. Es tal la cantidad de factores y agentes involucrados y tan intrincada su interrelación que se hace difícil explicar en términos sencillos las diferencias en los niveles observados en diferentes pruebas tales como PISA (OECD, 2016). Esto puede explicar la razón por la cual algunos intentos de adaptar parte o la totalidad de las metodologías de enseñanza de las matemáticas no han tenido el mismo conjunto de resultados exitosos que ellas han tenido en el país de origen.

En los últimos años, se ha relevado también el rol que tienen las emociones en el aprendizaje de las matemáticas. Tal como indica Pekrun (2014), el aula es un lugar de emociones, en donde los estudiantes pueden experimentar una serie de estados, como entusiasmo, admiración, empatía, envidia hacia los pares, aburrimiento, persistencia, etc., siendo probablemente algunos de esos estados moderados por su propio entorno familiar. También las creencias de los profesores(as) han relevado tener un rol importante a la hora de explicar algunos resultados (Maass y Schlöglmann, 2009; Mapolelo, y Akinsola, 2015). Así por ejemplo, las creencias, predisposiciones o preconcepciones que puedan tener los profesores(as) para preferir o desestimar incursionar en nuevas metodologías de enseñanza, en la manera en que facilitan o inhiben la autonomía por considerarla poco formal, o en la manera en que perciben y transmiten el saber matemático, tendrán efectos directos en el discurso didáctico, entendido éste como aquella disertación estructurada, en condiciones situacionales propicias, que interactúa a través del lenguaje como factor fundamental y que puede estructurar contingencias de índole intra, extra o transituacional (Ibañez,2007). Muy relacionado con lo anterior, hay evidencia de una relación positiva entre el autoconcepto matemático y el logro matemático, y las habilidades matemáticas automatizadas, lo cual está en línea con las expectativas basadas en estudios previos (Luo et al., 2014; Parker et al., 2013).

Las diferencias observadas en las puntuaciones de mediciones en matemáticas de los países en las pruebas internacionales, han sugerido buscar adaptar o profundizar en aspectos propios de las metodologías que utilizan, con el propósito de poder mejorar en los propios rendimientos. Sin embargo, la concepción metodológica desde el punto de vista teóricocurricular no es la única explicación del éxito de la metodología. Por ejemplo, dentro del 
algunas de las características del llamado Método Singapur (Kaur, 2010), se mencionan como elementos fundamentales el énfasis en la resolución de problemas por sobre la mecánica o los procedimientos o las fórmulas, la adquisición de habilidades y el desarrollo de buenos hábitos de pensamiento, el uso de diagramas y representaciones, aspectos que también recoge en parte el currículo finlandés (Kupari, 2008). Todos estos énfasis son además consistentes con teorías clásicas y ampliamente aceptadas como la del aprendizaje por descubrimiento de Bruner (1960), la variabilidad perceptual dentro de las condiciones para aprender matemáticas según Dienes (1969), y la comprensión instrumental relacionada con la psicología del aprendizaje de las Matemáticas de Skemp (1980). Sin embargo, una revisión de los objetivos y propósitos del Currículo Chileno (MINEDUC, 2012) muestran que las características anteriormente mencionadas para el método Singapur también están presentes en las Bases Curriculares, de la misma manera que en España se mencionan estos elementos en el currículo básico de la Educación Primaria, ESO y Bachillerato (Real Decreto 126/2014 del Ministerio de Educación, Cultura y Deporte, 2014). Este hecho ilustra que las comparaciones entre metodologías no pueden establecerse sólo comparando los currículos y sus objetivos, sino que se deben analizar otros aspectos importantes relacionados con el contexto, la implementación y dinámica permanente del quehacer pedagógico, como lo pueden ser, por ejemplo, el rol social del profesorado, y la estructura y secuenciación de su perfeccionamiento y praxis pedagógica, en donde efectivamente, Singapur tiene diferencias importantes frente a los mecanismos de soporte al hacer y quehacer pedagógico de los dos países de habla hispana antes mencionados (Kaur, 2010).

Adicionalmente, es importante relevar que en todos los juicios de valor anteriormente realizados hacia algunos de los factores que pueden incidir al juzgar la calidad de la enseñanza de las matemáticas, cabe plantearse la interrogante acerca de cómo se está midiendo la efectividad del proceso de enseñanza-aprendizaje, interrogante que da paso a otra área de investigación sobre la cual se han experimentado avances notables en los últimos años. Este tema reviste particular importancia porque tampoco hay consenso sobre cuál es la mejor manera de analizar la efectividad del proceso de enseñanza-aprendizaje. Por ejemplo, el promedio general de calificaciones puede considerarse como un parámetro cercano observable al alumno(a), pues recoge información por parte del profesor que interactúa directamente con él/ella. Sin embargo, y por lo mismo, este parámetro puede recoger también eventuales sesgos de género o interculturales, o ser consecuencia de procesos de evaluación que no estén totalmente alineados con el trabajo en el aula. Los instrumentos estandarizados, por otro lado, permiten un estudio poblacional amplio que podría explicar diferencias y establecer comparaciones entre categorías más generales. Sin embargo, por esta misma razón son muchas veces utilizados como parte de políticas públicas que incentivan o castigan sus resultados, pasando a distorsionar el propio proceso formativo, hecho advertido ya hace más de 15 años por Popham (2001) y posteriormente confirmado con mayor evidencia (Popham, 2008). Si bien dicho estudio indicaba expresamente que las pruebas bien diseñadas y utilizadas adecuadamente pueden ser de gran valor para la educación, también reconocía una serie de riesgos o inconvenientes, como por ejemplo, el que aspectos importantes del currículo se estén 
haciendo a un lado, porque no son medidos por las pruebas, que pasa a ser la prioridad para el establecimiento; que los niños terminen siendo entrenados intensamente para que dominen el contenido de esas pruebas, generando como consecuencia, una animadversión hacia la escuela, y que incluso los propios profesores dediquen su clase a preparar a sus alumnos para las pruebas, modificando el tiempo destinado para la enseñanza, con el propósito de aumentar las puntuaciones de los alumnos sin elevar su competencia en los aspectos que se supone miden las pruebas. Se hace necesario contar con estudios que puedan complementar ambos niveles de información, acogiendo además otros factores que permitan capturar de la manera más realista posible, el proceso de enseñanza y aprendizaje que está ocurriendo en el aula.

Este número especial de la revista pretende poner al alcance de la comunidad educativa y de la sociedad en general, en un mismo escenario, un conjunto de trabajos de ocho grupos de investigación, conformados por especialistas de diversas universidades europeas y latinoamericanas, de dilatada y reconocida trayectoria en el área de la investigación en educación matemática, de manera de hacer patente a través de su lectura, parte de la compleja trama de causas y efectos directos e indirectos que cada uno de los temas de estudio puede tener sobre los otros a la hora de intentar profundizar en sus conclusiones.

Los trabajos son de naturaleza diversa, y abarcan un amplio espectro de niveles y realidades educativas, y que son abordadas desde las matemáticas escolar de perspectivas investigativas cuali y cuantitativas, y con diseños de investigación descriptivos-correlacionales o cuasi experimentales. Las temáticas abordan temas asociados a la resolución de problemas, tanto como una posible estrategia de perfeccionamiento como desde el análisis del discurso de profesores acerca de su implementación. También, se aborda el estudio de diversas propuestas de intervención para el abordaje de niños y niñas con riesgo de presentar dificultades en el aprendizaje matemático, especialmente en ciclos educativos iniciales. Finalmente, se puede mencionar una temática que aborda un modelo complejo que examina variables de carácter predictivo asociadas al rendimiento escolar, o el examen de errores asociados a la resolución de diversas tareas que examinan competencias matemáticas tempranas. Todas ellas, de seguro entregarán a la comunidad educativa un conjunto de alternativas fundadas para que las instituciones educativas y el profesorado en general enfrente con mejor posibilidad de éxito el aprendizaje de las matemáticas, y puedan, por otra parte, identificar, contextualizar o incluso potenciar ciertas variables asociadas al rendimiento académico que deben ser antecedidas en ciclos iniciales.

La resolución de problemas es frecuentemente señalada como una alternativa fundamental para alcanzar mejores logros de aprendizaje en matemáticas. Los primeros dos artículos de este monográfico examinan propuestas en esta área, desde perspectivas y análisis diversos, pero que de seguro contribuyen de forma significativa al desarrollo del corpus teórico y empírico de esta metodología. La primera de ellas se desarrolla en el ámbito de la educación permanente, y se relaciona con una nueva metodología de perfeccionamiento para profesores de matemáticas en ejercicio. En este sentido, las propuestas y alternativas que se postulen, deben no sólo contar con una sólida fundamentación teórica y didáctica, sino también, con una base de información experimental que valide su pertinencia y real impacto. 
Es en este contexto que el primer artículo del monográfico, a cargo de un grupo de investigadores chilenos que se desempeñan en la Universidad de Chile y de Concepción, examina los resultados del efecto de un taller de desarrollo profesional basado en la resolución de problemas en las concepciones acerca de la naturaleza, logros y aprendizaje en matemáticas de los docentes chilenos de educación primaria (Cerda et al, 2017). La implementación de estrategia, examinada con un diseño cuasi-experimental, con pre y post prueba, permitió atenuar de forma significativa las creencias del profesorado que dejan entrever una concepción formalista de las matemáticas como un proceso rígido y estructurado. La participación de los profesores en el taller disminuyó su necesidad de protagonizar el aprendizaje, aumentando la valoración de prácticas de resolución de problemas centradas en el estudiante. Conjuntamente, los profesores participantes disminuyeron de forma significativa la visión del acceso al aprendizaje de las matemáticas como una condición fija o prácticamente inalterable, asociada más bien con capacidades innatas de los estudiantes o con estereotipos de género o étnicos. Finalmente, la estrategia fue altamente valorada por los docentes, quienes además reportaron mejoras en la percepción sobre su competencia y autoeficacia para implementar y transferir esta estrategia en el aula con sus estudiantes.

El segundo artículo resume la investigación de dos reconocidos investigadores de la Universidad de Salamanca, quienes realizan un sólido análisis del discurso motivacional de profesores de educación primaria durante la resolución de problemas matemáticos en el aula (De Sixte, y Rosales, 2017). La investigación revela que los esfuerzos de los profesores se orientan predominantemente a la realización de la actividad propiamente tal, acentuando la persistencia y el esfuerzo para acometer y resolver el problema por parte del estudiante, aun cuando ellos mismos expresan poner énfasis en el desarrollo de la motivación intrínseca y las creencias de competencia en el alumnado. El análisis muestra la inexistencia de acciones tendientes a mostrar dicha tarea como intrínsecamente motivante, o que las mismas persigan la búsqueda del logro desde la maestría o el aprendizaje. Esta evidencia parece plantear una reflexión más exhaustiva de los discursos del profesorado y la necesidad de contrastarlos de forma efectiva con su praxis de aula.

En la línea de analizar diversas propuestas, modalidades y estrategias de intervención para atender y mejorar el logro de niños y niñas que presentan riesgo o dificultades en al aprendizaje de las matemáticas, emerge con propiedad el tercer artículo del grupo de investigación español de la Universidad de La Laguna, cuyo propósito fue analizar los efectos sobre el conocimiento y valoración de un sistema de aprendizaje tutorial online en profesorado tutor y de apoyo en las Islas Canarias para la instrucción temprana de las matemáticas que atienden niños y niñas en riesgo de presentar Dificultades Específicas de Aprendizaje en Matemáticas (DEAM) (Jiménez, y Gutiérrez, 2017). El trabajo reporta los efectos del programa tutorial Primate sobre los conocimientos y la valoración que realiza el profesorado tutor y el profesorado de apoyo en las Islas Canarias. La propuesta de aprendizaje tutorial sobre la base de plataforma Moodle, mostró una valoración positiva de su potencial en ambos grupos de profesores, y del mismo modo, ambos grupos mejoraron sus conocimientos en las distintas áreas de conocimiento evaluadas a través de dicho sistema tutorial. Toda la evidencia 
presentada en el trabajo, muestra que una propuesta de naturaleza virtual puede constituir una alternativa real al fortalecimiento de competencias y conocimientos del profesorado que atiende niños y niñas con DEAM.

En el ámbito de las propuestas de abordaje de estudiantes con dificultades de aprendizaje de las matemáticas, el cuarto artículo pertenece a un grupo de investigadores de la Universidad de Cádiz, y presenta el impacto sobre las habilidades matemáticas tempranas producto de la implementación de una nueva metodología de enseñanza de las matemáticas, denominada Método Abierto Basado en Números (Método ABN) y que se está adoptando oficialmente en la comunidad autónoma de Andalucía (Aragón, Delgado, y Marchena, 2017). Esta metodología se caracteriza por ser flexible, transparente y contextualizada, resultando muy adecuada para prevenir dificultades de aprendizaje de las matemáticas, en donde se requiere precisamente el emplear metodologías de enseñanza que se adapten al nivel y al ritmo de aprendizaje de dichos alumnos. Se analizó los efectos de la metodología ABN frente a la metodología tradicional, mostrando diferencias significativas en los niveles de ganancias en los distintos componentes evaluados, especialmente en el ámbito relacional, y en las habilidades numéricas de estimación y conocimiento general de los números.

En este mismo dominio, se presenta quinto artículo del grupo de investigación del Departamento de Psicología de la Universidad de Cádiz, una propuesta de intervención para estudiantes de educación primaria en riesgo de presentar dificultades en el aprendizaje de las matemáticas (Navarro, Navarro, y Menacho, 2017). Un grupo de estos estudiantes presentaron mejoras significativas en los componentes asociados al dominio general y específico: nueve dimensiones de competencias matemáticas tempranas, cuatro del dominio lógico relacional y cinco de tipo numéricas numérico, y, también en aspectos de la memoria de trabajo, al ser expuestos a un programa de entrenamiento matemático basado en actividades de instrucción directa. El programa de entrenamiento individualizado y las actividades propuestas, emerge como una alternativa de prevención efectiva para este tipo de alumnos.

El análisis del logro o fracaso en matemáticas, demuestra que este es un fenómeno multifactorial, que tiene muchas veces relaciones de interacción e interdependencia mutua. A través de este tipo de análisis se pueden determinar algunas variables que pueden relacionarse de forma significativa con buenos aprendizajes o bien pueden predecir el fracaso o dificultades en el aprendizaje matemático escolar. En este contexto se analizan los resultados del sexto artículo de este monográfico, que examina la relación entre variables de naturaleza cognitivoafectivas y el nivel de logro en un grupo de estudiantes secundarios holandeses de 12 a 14 años (Timmerman, Toll, y Van Luit, 2017). Se logró constatar una asociación positiva entre el autoconcepto matemático y los resultados en matemáticas en los cuatro dominios evaluados (medidas, relaciones, numeración y escalas), así como con las habilidades matemáticas automatizadas. Igualmente, se encontraron correlaciones negativas significativas entre la ansiedad ante las matemáticas y los resultados en esta materia para las tareas de escalas. El estudio pone de manifiesto que, en el escenario escolar, es fundamental que los profesores y, porque no decirlo, la familia y la comunidad toda, atienda y estimule creencias y sentimientos positivos de los estudiantes sobre su propia competencia en matemáticas, pues de esa forma se 
puede mejorar el rendimiento en esta materia.

Del mismo modo, en esta línea de factores asociados al rendimiento académico en matemáticas, no se pude soslayar el rol de las creencias epistemológicas que poseen los estudiantes acerca de las matemáticas. En el séptimo artículo de este número especial se presentan los resultados de la investigación de Vizcaino y Manzano (2017), dos investigadoras de la Universidades cubanas, las que examinan las creencias epistemológicas acerca de las matemáticas, derivados de los trabajos de Schoemmer y Duell (2013). El análisis muestra que estas creencias del estudiante guardan relación estrecha con el logro y rendimiento en matemáticas. Más aún, si los estudiantes poseen creencias denominadas poco productivas acerca de ella, como pensar que su aprendizaje no requiere esfuerzo personal o que es una habilidad innata, o creen en la simplicidad de este conocimiento, sus logros son claramente más bajos. En cambio, si ese conocimiento es percibido como relativo su rendimiento es más favorable.

Bajo un enfoque complementario al anterior, en la búsqueda de variables o factores que tengan el carácter de predictores del desempeño o dificultades futuras en matemáticas que los estudiantes puedan presentar en su itinerario escolar, el trabajo acerca de los marcadores nucleares de la competencia aritmética en preescolares, liderado por un grupo de investigadores liderado por José Orrantia, del Departamento de Psicología Evolutiva y de la Educación de la Universidad de Salamanca, y también integrado por otros dos investigadores, del Instituto Nacional de Educación de Nanyang, Singapur, y de la Universidad de Leuven, Bélgica (Orrantia et al., 2017), constituye el octavo artículo de este número especial. La investigación busca comprobar si las variables de procesamiento numérico predicen más allá de las variables de control, como inteligencia, velocidad de procesamiento, amplitud de memoria, control inhibitorio y memoria visuo-espacial. El modelo predictivo analizado mostró que la comparación de magnitudes simbólicas y la enumeración contribuyeron a la varianza en ejecución aritmética más allá de las variables de control, mientras que la comparación de magnitudes no simbólicas no contribuyó significativamente. Estos hallazgos, relevan que los profesores deben poner énfasis en el desarrollo de un buen conocimiento de los números simbólicos, ya que resulta importante para el desarrollo matemático futuro de los niños, y que, del mismo modo, la representación de la magnitud puede ser crucial para el desarrollo aritmético.

El noveno artículo corresponde a un análisis en la población italiana, se examinan los errores cometidos por los alumnos al resolver cada una de las tareas de tipo relacional: comparación, clasificación, correspondencia y seriación, del ámbito lógico-relacional, y aquellos cometidos en tareas del ámbito numérico, como conteo verbal, conteo estructurado, conteo resultante, conocimiento general de los números y estimación. A través del registro, observación e interpretación de las estrategias utilizadas por los niños para resolver dichas tareas, se postula romper la barrera del error como sinónimo de fracaso y avanzar hacia mejores estrategias de potenciamiento y prevención de las dificultades de aprendizaje (González, Benvenutto, y Lanciano, 2017).

El artículo final aborda una línea de trabajo emergente y aun poco explorada respecto 
del aprendizaje y logro en matemáticas, que se relaciona con el clima de aula, o el ambiente en el cual se desarrolla la clase. En este caso los investigadores de la Universidad de Concepción, O’Higgins y de la Universidad de Córdoba, examinan el impacto de la percepción sobre diversos aspectos de la convivencia escolar y su rol mediador en el rendimiento académico, a través del estudio de aspectos tales como la gestión interpersonal, red social de iguales, agresión, victimización, disruptividad, indisciplina y desidia escolar, al interior de la escuela o institución escolar (Salazar et al., 2017). La investigación releva el rol negativo que presenta la percepción de ocurrencia de situaciones de indisciplina e involucramiento en actos de agresión, respecto del promedio de calificaciones en matemáticas, especialmente en el grupo de las chicas y en contextos de mayor vulnerabilidad social.

En síntesis, a pesar de la aparente particularidad de cada uno de los artículos presentados, todos ellos pueden ser entendidos como parte del esfuerzo de la comunidad científica para incursionar en el complejo desafío del entendimiento global del proceso de enseñanza y aprendizaje de las matemáticas: la formación del profesorado, las dificultades en el aprendizaje de las matemáticas, las nuevas tecnologías y nuevas metodologías, el análisis de los errores y el análisis de las relaciones entre factores afectivos y cognitivos, dan cuenta que probablemente no exista en el futuro inmediato una respuesta completa para mejorar los niveles de logro esperados en matemáticas, pero su lectura deja entrever que la búsqueda de dicha respuesta pasa necesariamente por conocer e integrar los resultados de las diversas manifestaciones del proceso de enseñanza y aprendizaje de esta hermosa y relevante disciplina.

\section{Referencias}

Aragón, E., Delgado, C., y Marchena, E. (2017). La Metodología ABN y el aprendizaje de las habilidades matemáticas tempranas. Psychology, Society \& Education, 9, 61-70.

Bruner, J. (1960). El Proceso de la Educación. México: Editorial Hispano Americana.

Cerda, G., Salazar, Y., Sáez, K., Casas, J., Ortega, R., y Pérez, C. (2017). Impacto de la percepción de los estudiantes respecto de la convivencia escolar sobre su rendimiento académico en Matemática. Psychology, Society \& Education, 147-161.

Cerda, G., Pérez, C., Giaconni, V., Perdomo-Díaz, J., Reyes, C., y Felmer, P., (2017). The effect of a professional development program workshop about problem solving on mathematics teachers' ideas about the nature of mathematics, achievements in mathematics, and learning in mathematics. Psychology, Society \& Education, 9, 11-26.

De Sixte, R., y Rosales, J. (2017). Análisis del discurso motivacional durante la resolución de problemas matemáticos en el aula. Psychology, Society \& Education, 9, 27-43.

Dienes, Z. (1969). Los Primeros Pasos en Matemática. Lógica y juegos lógicos. Barcelona: Editorial Teide.

González, I., Benvenutto, G., y Lanciano, N. (2017). Dificultades de Aprendizaje en Matemática en los niveles iniciales: Investigación y formación en la escuela italiana. Psychology, Society \& Education, 9, 135-145.

Ibáñez, C. (2007). Un análisis crítico del modelo del triángulo pedagógico. Una propuesta 
alternativa. Revista Mexicana de Investigación Educativa, 12(32), 435-456.

Jiménez, J. E., y Gutiérez, N. (2017). Efectos y valoración de un sistema de aprendizaje tutorial online en profesorado de las Islas Canarias para la instrucción temprana de las matemáticas en población de riesgo. Psychology, Society \& Education, 9, 45-59.

Kaur, B. (2010). Towards Excellence in Mathematics Education-Singapore's Experience. Procedia - Social and Behavioral Sciences 8, 28-34.

Kupari, P. (2008). Mathematics education in Finnish comprehensive school: Characteristics contributing to student success. Proceedings of the XI International Congress in Mathematics Education, ICME. México.

Luo, W., Hogan, D., Tan, L. S., Kaur, B., Ng, P. T., \& Chan, M. (2014). Self-construal and students' math self-concept, anxiety and achievement: An examination of achievement goals as mediators. Asian Journal of Social Psychology, 17, 184-195. doi:10.1111/ajsp.12058

Maass, J. and Schlöglmann, W. (2009). Beliefs and Attitudes in Mathematics Education. New Research Results. Sense Publishers, Rotterdam.

Mapolelo, D. and Akinsola, M. (2015). Preparation of Mathematics Teachers: Lessons from Review of Literature on Teachers' Knowledge, Beliefs, and Teacher Education. American Journal of Educational Research, 3(4), pp. 505-513. DOI: 10.12691/education-3-4-18.

MINEDUC (2012). Bases Curriculares para la Educación Básica. Unidad de Currículum y Evaluación, Ministerio de Educación, República de Chile.

Ministerio de Educación, Cultura y Deporte (2014). Real Decreto 126/2014. España.

Navarro, J. I., Navarro, M. J., y Menacho, I. (2017). Instrucción directa con alumnado de Educación Primaria en riesgo de dificultades en el aprendizaje de las matemáticas. Psychology, Society \& Education, $n$ (vol), pp.

OECD (2016). PISA 2015 Results in Focus. Accedido el 1 de marzo 2017 en https://www.oecd.org/pisa/pisa-2015-results-in-focus.pdf

Orrantia, J., San Romualdo, S., Matilla, L., Sánchez, M. R., Múñez, D., y Verschaffel, L. (2017). Marcadores nucleares de la competencia aritmética en preescolares. Psychology, Society \& Education, 9, 121-134.

Parker, P. D., Marsh, H. W., Ciarrochi, J., Marshall, S., \& Abduljabbar, A. S. (2013). Juxtaposing math self-efficacy and self-concept as predictors of long-term achievement outcomes. Educational Psychology, 34, 29-48. doi :10.1080/01443410.2013.797339

Pekrun, Reinhard (2014). Emotions and learning. [Educational practices series-24].Ginebra: The international Bureau of Education-UNESCO. Consultado online el 01 de marzo de 2017 en el sitio http://www.ibe.unesco.org/fileadmin/user_upload/Publications/Educational_Practices/E dPractices_24eng.pdf

Popham, W. J. (2001). Frontline: testing our schools: interviews: James Popham. Consultado $\begin{array}{lllll}\text { el } & 1 & \text { de } & 2017 & \text { en: }\end{array}$ http://www.pbs.org/wgbh/pages/frontline/shows/schools/interviews/popham.html 
Popham, W. J. (2008). Standards-based education: Two wrongs don't make a right. En S. Mathison y E. W. Ross, eds. The nature and limits of standards-based reform and assessment (pp. 15-25). Nueva York: Teachers College Press, pp. 15-25.

Schommer, M., \&Duell, O. K. (2013). Domain Specific and General Epistemological Beliefs. Their Effectson Mathematics. Revista de Investigación Educativa, 31(2), 317-330. doi.org/10.6018/rie.31.2.170911

Skemp, R. (1980). Psicología del Aprendizaje de las Matemáticas. Madrid: Ediciones Morata.

Timmerman, H. L., Toll, S., \& Van Luit, J. (2017). The relation between math self-concept, test and math anxiety, achievement motivation and math achievement in 12 to 14-yearold typically developing adolescents. Psychology, Society \& Education, 9, 89-103.

Vizcaino, A.E., y Manzano, M. (2017). Análisis de las relaciones entre creencias epistemológicas sobre las matemáticas y rendimiento académico. Psychology, Society \& Education, 9, 105-119. 